\title{
Generic points for dynamical systems with average shadowing
}

\author{
Dominik Kwietniak $^{1,2}$ (D) Martha Lacka ${ }^{1}$. \\ Piotr Oprocha ${ }^{3,4}$
}

Received: 17 April 2016 / Accepted: 3 November 2016 / Published online: 14 November 2016 (C) The Author(s) 2016. This article is published with open access at Springerlink.com

\begin{abstract}
We study the Besicovitch pseudometric $D_{B}$ for compact dynamical systems. The set of generic points of ergodic measures turns out to be closed with respect to $D_{B}$. It is proved that the weak specification property implies the average asymptotic shadowing property and the latter property does not imply the former one nor the almost specification property. Furthermore an example of a proximal system with the average shadowing property is constructed. It is proved that to every invariant measure $\mu$ of a compact dynamical system one can associate a certain asymptotic pseudo orbit such that any point asymptotically tracing in average that pseudo orbit is generic for
\end{abstract}

Communicated by H. Bruin.

$凶$ Dominik Kwietniak

dominik.kwietniak@uj.edu.pl

http://www.im.uj.edu.pl/DominikKwietniak/

Martha Łącka

martha.lacka@doctoral.uj.edu.pl

http://www2.im.uj.edu.pl/MarthaLacka/

Piotr Oprocha

oprocha@agh.edu.pl

1 Faculty of Mathematics and Computer Science, Jagiellonian University in Krakáw, ul. Łojasiewicza 6, 30-348 Kraków, Poland

2 Institute of Mathematics, Federal University of Rio de Janeiro, Av. Athos da Silveira Ramos 149, Cidade Universitaria - Ilha do Fundão, Rio de Janeiro 21945-909, Brazil

3 Faculty of Applied Mathematics, AGH University of Science and Technology, al. Mickiewicza 30, 30-059 Kraków, Poland

4 National Supercomputing Centre IT4Innovations, Division of the University of Ostrava, Institute for Research and Applications of Fuzzy Modeling, 30. Dubna 22, 70103 Ostrava, Czech Republic 
$\mu$. A simple consequence of the theory presented is that every invariant measure has a generic point in a system with the asymptotic average shadowing property.

Keywords Generic points - The asymptotic average shadowing property · Dbar pseudometric

Mathematics Subject Classification 37A05 $\cdot$ 37B05 $\cdot$ 37B10

\section{Introduction}

The specification property or one of its variants are often applied in the theory of dynamical systems ${ }^{1}$ to construct invariant measures with special properties (for example, measures of maximal entropy or equilibrium states, see $[6,9])$. These properties also help to define points with predetermined statistical behaviour like generic points or "non-typical" points, see [4,9]. Here, using the Besicovitch pseudometric, we show how to construct a certain asymptotic pseudo orbit associated with a given measure $\mu$. This generalizes several proofs of existence of generic points for a given invariant measure, provided that a sufficiently strong tracing method is available in the system, e.g. see [7,8,31-34].

The Besicovitch pseudodistance measures the average separation of points along two sequences in the space of all infinite $X$-valued sequences $X^{\infty}=\left\{\left\{x_{n}\right\}_{n=0}^{\infty}: x_{n} \in\right.$ $X\}$ and is given for $\underline{x}=\left\{x_{n}\right\}_{n=0}^{\infty}, \underline{x}^{\prime}=\left\{x_{n}^{\prime}\right\}_{n=0}^{\infty} \in X^{\infty}$ by

$$
D_{B}\left(\underline{x}, \underline{x}^{\prime}\right)=\limsup _{N \rightarrow \infty} \frac{1}{N} \sum_{n=0}^{N-1} \rho\left(x_{n}, x_{n}^{\prime}\right)
$$

It extends naturally to a pseudometric on $X$ given by the average separation of points along orbits of a dynamical system $(X, T)$, that is,

$$
D_{B}(x, y)=\limsup _{N \rightarrow \infty} \frac{1}{N} \sum_{n=0}^{N-1} \rho\left(T^{n}(x), T^{n}(y)\right),
$$

where $x, y \in X$ and $\rho$ is a metric on $X$. An equivalent pseudometric is well known in the ergodic theory and symbolic dynamics as $\bar{d}$-pseudometric, see [30,37]. A similar Weyl pseudodistance and the accompanying notion of quasi-unifrom convergence were also widely studied, see [13,18].

We prove that a point is generic for a non-necessarily ergodic measure if it asymptotically traces a special approximate trajectory (which in turn is a variant of the average asymptotic pseudoorbit), where the Besicovitch pseudodistance is used to define the pseudotrajectory and measures the accuracy of tracing. Recall that $x \in X$ is generic for $\mu$ if

\footnotetext{
${ }^{1}$ By a dynamical system we mean a pair $(X, T)$, where $X$ is a compact metric space and $T$ is a continuous map from $X$ to itself.
} 


$$
\lim _{n \rightarrow \infty} \frac{1}{n} \sum_{j=0}^{n-1} f\left(T^{j}(x)\right)=\int_{X} f \mathrm{~d} \mu \text { for every continuous } f: X \rightarrow \mathbb{R}
$$

As a corollary we get that if a system has the asymptotic average shadowing property (a notion introduced by Gu in [17], see below), then every invariant measure has a generic point, because in such a system every average asymptotic pseudo-orbit is followed by the orbit of some point. The same result is proved independently in [10] via a direct construction. We believe that using the Besicovitch pseudodistance provides a new perspective and leads to short proofs extending the theory (see the proof of Theorem 21 and Example 23).

In order to compare our result with earlier developments, recall that generic points have full measure for every ergodic invariant measure, but non-ergodic invariant measures need not to have generic points. Sigmund proved that if a dynamical system has the periodic specification property (see [32]), then every invariant measure has a generic point. Many authors generalized this result.

We show that yet another variant of specification, known as the weak specification property implies the asymptotic average shadowing property. This together with results of [19] gives us the following diagram illustrating the connections between main generalizations of specification (we refer the reader to [22] for more details; here "AASP" stands for "(the) asymptotic average shadowing property"):

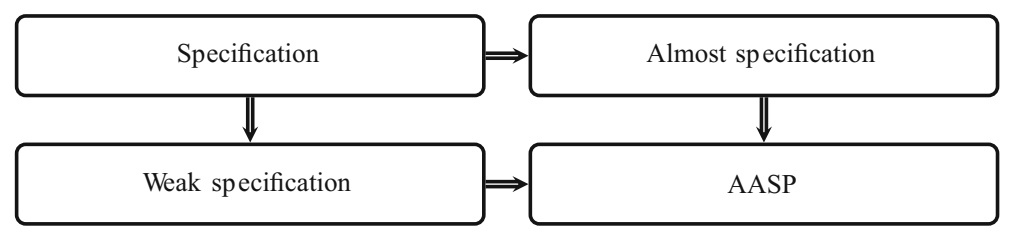

It is proved in [23] that there are systems with the weak specification property, but without the almost specification property, hence there are systems with the asymptotic average shadowing property, but without almost specification property. This answers a problem we considered in [19]. We finish the paper with an example demonstrating that there are proximal systems with the average shadowing property, which also answers some natural questions one may ask about properties of systems with a generalized shadowing.

This paper is organized as follows. In Sect. 2 we recall some standard definitions from topological dynamics and related fields. Section 3 discusses the definition and basic properties of the Besicovitch pseudometric. In Sect. 4 we show that a set of measures generated by an orbit varies continuously as a function from $X$ equipped with the Besicovitch pseudometric to the space of compact subsets of invariant measures considered with the Hausdorff distance. It follows that the set of generic points of ergodic measures is a closed set with respect to the Besicovitch pseudodistance. Section 5 establishes the relation between weak specification property and asymptotic average shadowing property. Possible applications of our studies on Besicovitch pseudodistance are provided in Sect. 6. Section 7 contains an example of a proximal, mixing shift space exhibiting the average shadowing property, but neither almost nor weak specification. 


\section{Topological dynamics}

\section{Notation}

Throughout this paper a pair $(X, T)$ is a dynamical system. This means that $X$ is a compact metric space and $T: X \rightarrow X$ is a continuous map. All properties of $T$ considered here are invariants of topological conjugacy, hence they do not depend on the choice of metric. Therefore we fix a metric $\rho$ for $X$ and assume without loss of generality that the diameter of $X$ is equal to 1 .

Given a compact metric space $Y$ and $A \subset Y$ we denote by $\operatorname{dist}(y, A)$ the distance of a point $y \in Y$ to the set $A$. For $\varepsilon>0$ we write $A^{\varepsilon}$ for the $\varepsilon$-hull of $A$, that is the set $\{y \in X: \operatorname{dist}(y, A)<\varepsilon\}$. Let $2^{Y}=\{K \subset Y: K$ compact and $K \neq \emptyset\}$ and $H_{Y}: 2^{Y} \times 2^{Y} \rightarrow \mathbb{R}_{+}$be the Hausdorff metric on $2^{Y}$ given by

$$
H_{Y}(A, B)=\max \left\{\inf \left\{\varepsilon>0: B \subset A^{\varepsilon}\right\}, \inf \left\{\varepsilon>0: A \subset B^{\varepsilon}\right\}\right\} .
$$

The upper asymptotic density of $A \subset \mathbb{N}_{0}$ is as usual denoted by $\bar{d}(A)$ and given by

$$
\bar{d}(A)=\limsup _{n \rightarrow \infty} \frac{\#(A \cap\{0,1, \ldots, n-1\})}{n} .
$$

If the above upper limit is in fact a limit, we denote it by $d(A)$ and call the asymptotic density of $A$.

\section{Recurrence properties}

Recall that $T$ is transitive if for all non-empty open sets $U, V \subset X$ there exists $n \geq 0$ such that $T^{n}(U) \cap V \neq \varnothing$. We say that $T$ is (topologically) mixing if for all nonempty open sets $U, V \subset X$ there exists $N \geq 0$ such that for all $n \geq N$ one has $T^{n}(U) \cap V \neq \varnothing$. A map $T$ is (topologically) weakly mixing if the product map $T \times T$ is transitive. Finally, $T$ is chain mixing if for every $\delta>0$ and all $x, y \in X$ there exists $N \in \mathbb{N}$ such that for every $n \geq N$ there is a finite sequence $x_{0}, x_{1}, \ldots, x_{n}$ in $X$ satisfying $x_{0}=x, x_{n}=y$, and $\rho\left(T\left(x_{i-1}\right), x_{i}\right)<\delta$ for $i=1, \ldots, n$.

\section{Average shadowing}

A sequence $\left\{x_{n}\right\}_{n=0}^{\infty} \in X^{\infty}$ is a $\delta$-average pseudoorbit for $T$ if there is an integer $N_{0}>0$ such that for every $N>N_{0}$ and $k \geq 0$ one has

$$
\frac{1}{N} \sum_{n=0}^{N-1} \rho\left(T\left(x_{n+k}\right), x_{n+k+1}\right)<\delta .
$$

A sequence $\left\{x_{n}\right\}_{n=0}^{\infty} \in X^{\infty}$ is an asymptotic average pseudoorbit for $T$ if

$$
\lim _{N \rightarrow \infty} \frac{1}{N} \sum_{n=0}^{N-1} \rho\left(T\left(x_{n}\right), x_{n+1}\right)=0 .
$$


Given $\varepsilon>0$ we say that a sequence $\left\{x_{j}\right\}_{j=0}^{\infty}$ is $\varepsilon$-shadowed in average by a point $x \in X$ if

$$
\limsup _{N \rightarrow \infty} \frac{1}{N} \sum_{n=0}^{N-1} \rho\left(T^{n}(x), x_{n}\right)<\varepsilon
$$

We say that $\left\{x_{n}\right\}_{n=0}^{\infty} \in X^{\infty}$ is asymptotically shadowed in average by $x \in X$ if

$$
\lim _{N \rightarrow \infty} \frac{1}{N} \sum_{n=0}^{N-1} \rho\left(T^{n}(x), x_{n}\right)=0 .
$$

A dynamical system $(X, T)$ has the average shadowing property if for every $\varepsilon>0$ there exists $\delta>0$ such that every $\delta$-average pseudo-orbit is $\varepsilon$-shadowed in average by some point. A system $(X, T)$ has the asymptotic average shadowing property if every asymptotic average pseudo-orbit of $T$ is asymptotically shadowed in average by some point.

\section{Weak specification}

An orbit segment of a point $x$ over an interval $[a, b]$, where $a \leq b$ are nonnegative integers is the sequence

$$
T^{[a, b]}(x)=\left(T^{a}(x), T^{a+1}(x), \ldots, T^{b-1}(x), T^{b}(x)\right) .
$$

Let $N: \mathbb{N} \rightarrow \mathbb{N}$ be any function. A family of orbit segments $\xi=\left\{T^{\left[a_{j}, b_{j}\right]}\left(x_{j}\right)\right\}_{j=1}^{n}$ is an $N$-spaced specification if $a_{i}-b_{i-1} \geq N\left(b_{i}-a_{i}+1\right)$ for $2 \leq i \leq n$. A specification $\xi=\left\{T^{\left[a_{j}, b_{j}\right]}\left(x_{j}\right)\right\}_{j=1}^{n}$ is $\varepsilon$-traced by $y \in X$ if

$$
\rho\left(T^{k}(y), T^{k}\left(x_{i}\right)\right) \leq \varepsilon \text { for } a_{i} \leq k \leq b_{i} \text { and } 1 \leq i \leq n .
$$

A dynamical system $(X, T)$ has the weak specification property if for every $\varepsilon>0$ there is a nondecreasing function $M_{\varepsilon}: \mathbb{N} \rightarrow \mathbb{N}$ with $M_{\varepsilon}(n) / n \rightarrow 0$ as $n \rightarrow \infty$ such that any $M_{\varepsilon}$-spaced specification is $\varepsilon$-traced by some point in $X$. We refer the reader to [22] for more information about specification.

\section{Invariant measures}

Let $\mathcal{M}(X)$ be the set of all Borel probability measures on $X$. Let $\vec{d}: \mathcal{M}(X) \times$ $\mathcal{M}(X) \rightarrow \mathbb{R}_{+}$be the Prokhorov metric, that is let

$$
\vec{d}(\mu, v)=\inf \left\{\varepsilon>0: \mu(B) \leq v\left(B^{\varepsilon}\right)+\varepsilon \text { for every Borel set } B \subset X\right\} .
$$

It is well known that the topology induced by $\vec{d}$ is the weak* topology on $\mathcal{M}(X)$. A measure $\mu \in \mathcal{M}(X)$ is: 
- $T$-invariant, if $\mu\left(T^{-1}(B)\right)=\mu(B)$ for every Borel set $B \subset X$,

- ergodic, if it is $T$-invariant and for every Borel set $B \subset X$ with $T^{-1}(B)=B$ either $\mu(B)=0$ or $\mu(B)=1$.

We denote by $\mathcal{M}_{T}(X) \subset \mathcal{M}(X)$ the family of all $T$-invariant measures and by $\mathcal{M}_{T}^{e}(X)$ the family of all ergodic measures. The measure center of $(X, T)$ is the smallest closed subset $F$ of $X$ such that $\mu(F)=1$ for every $T$-invariant measure $\mu$.

For $x \in X$ let $\hat{\delta}(x) \in \mathcal{M}(X)$ be the Dirac measure supported on $\{x\}$. Given $\underline{x}=\left\{x_{j}\right\}_{j=0}^{\infty} \in X^{\infty}$ and $n \in \mathbb{N}$ we define an empirical measure:

$$
\mathfrak{m}(\underline{x}, n)=\frac{1}{n} \sum_{i=0}^{n-1} \hat{\delta}\left(x_{i}\right) .
$$

For $x \in X$ let $\mathfrak{m}_{T}(x, n)$ denote the measure $\mathfrak{m}\left(\left\{T^{j}(x)\right\}_{j=0}^{\infty}, n\right)$. If $T$ is clear from the context, then we write $\mathfrak{m}(x, n)$ for $\mathfrak{m}_{T}(x, n)$. We call such a measure an empirical measure of $x$. A point $x \in X$ is called generic for $\mu \in \mathcal{M}(X)$ if the sequence $\left\{\mathfrak{m}_{T}(x, n)\right\}_{n=0}^{\infty}$ converges to $\mu$ in the weak* topology. The set of generic points of $\mu$ is denoted $\operatorname{Gen}(\mu)$.

A measure $\mu \in \mathcal{M}(X)$ is a distribution measure for a sequence $\underline{x}=\left\{x_{n}\right\}_{n=0}^{\infty} \in X^{\infty}$ if $\mu$ is a limit of some subsequence of $\{\mathfrak{m}(\underline{x}, n)\}_{n=1}^{\infty}$. The set of all distribution measures of a sequence $\underline{x}$ is denoted by $\hat{\omega}(\underline{x})$. Clearly, $\hat{\omega}(\underline{x})$ is a closed and nonempty subset of $\mathcal{M}(X)$ (since $\overline{\mathcal{M}}(X)$ is compact), it is also connected, because $\vec{d}(\mathfrak{m}(\underline{x}, n), \mathfrak{m}(\underline{x}, n+$ 1)) $\rightarrow 0$ as $n \rightarrow \infty$.

\section{Symbolic dynamics}

Let $\mathcal{A}$ be a finite set of symbols with discrete topology. Let $\mathcal{A}^{\infty}$ be a product topological spaces. By $\sigma$ we denote the continuous onto map from $\mathcal{A}^{\infty}$ to itself given by $\sigma(\omega)_{i}=$ $\omega_{i+1}$, where $\omega=\left(\omega_{i}\right) \in \mathcal{A}^{\infty}$. A shift space is a nonempty, closed, and $\sigma$-invariant subset of $\mathcal{A}^{\infty}$. For more details on symbolic dynamics we refer to [25].

\section{Besicovitch pseudometric}

To elucidate the construction of generic points we introduce the Besicovitch pseudometric. Recall that a pseudometric on $X$ is a function $p: X \times X \rightarrow[0, \infty)$ which is symmetric, obeys the triangle inequality, and $p(x, x)=0$ for all $x \in X$. Every pseudometric space is a topological space in a natural manner, and most notions defined for metric spaces, like the (uniform) equivalence of metrics, remain meaningful for pseudometric spaces.

Recall that $X^{\infty}$ denotes the set of all sequences indexed with nonnegative integers with entries in $X$. Given $T: X \rightarrow X$ we can define a map (also denoted by $T$ ) on $X^{\infty}$ by $T(\underline{x})=\left\{T\left(x_{n}\right)\right\}_{n=0}^{\infty}$ for any $\underline{x}=\left\{x_{n}\right\}_{n=0}^{\infty} \in X^{\infty}$. We define the shift map on $X^{\infty}$ by $\sigma(\underline{x})_{n}=x_{n+1}$ for $n \in \mathbb{N}_{0}$ and $\underline{x}=\left\{x_{n}\right\}_{n=0}^{\infty} \in X^{\infty}$.

We can restate the definition of a pseudo orbit in our notation as follows: a sequence $\underline{x}=\left\{x_{n}\right\}_{n=0}^{\infty} \in X^{\infty}$ is a $\delta$-pseudo orbit if $D_{\text {sup }}(T(\underline{x}), \sigma(\underline{x}))<\delta$, where $D_{\text {sup }}$ denotes 
the supremum metric on $X^{\infty}$. The orbit of $y \in X$ is said to $\varepsilon$-trace $x \in X^{\infty}$ if the sequence of $\left\{T^{n}(y)\right\}_{n=0}^{\infty} \in X^{\infty}$ is $\varepsilon$-close to $\underline{x}$ in the $D_{\text {sup }}$ sense. It turns out that in order to construct invariant measures and generic points it is convenient to work with the following pseudometric on $X^{\infty}$.

Definition 1 The Besicovitch pseudometric $D_{B}$ on $X^{\infty}$ is defined for $\underline{x}=\left\{x_{n}\right\}_{n=0}^{\infty}$, $\underline{x}^{\prime}=\left\{x_{n}^{\prime}\right\}_{n=0}^{\infty} \in X^{\infty}$ by

$$
D_{B}\left(\underline{x}, \underline{x}^{\prime}\right)=\limsup _{N \rightarrow \infty} \frac{1}{N} \sum_{n=0}^{N-1} \rho\left(x_{n}, x_{n}^{\prime}\right) .
$$

The Besicovitch pseudometric on $(X, T)$, also denoted by $D_{B}$, equals the $D_{B}$-distance between orbits, that is,

$$
D_{B}\left(x, x^{\prime}\right)=D_{B}\left(\left\{T^{n}(x)\right\}_{n=0}^{\infty},\left\{T^{n}\left(x^{\prime}\right)\right\}_{n=0}^{\infty}\right) .
$$

It is easy to see that these functions are indeed pseudometrics on $X^{\infty}$ and $X$, respectively. Furthermore, $D_{B}$ is $\sigma$-invariant on $X^{\infty}$ and $T$-invariant on $X$. Besicovitch used a similar pseudodistance in his study of almost periodic functions, but the idea of measuring the average distance along two orbits in a dynamical systems is so natural that probably it has been reinvented independently by many authors, see Aulsander [2] who mentions earlier works of Fomin and Oxtoby, or [5,11,12,15,28] for more recent applications.

The pseudometric $D_{B}$ depends on $\rho$ but this is not reflected in our notation because we will show that equivalent metrics induce uniformly equivalent Besicovitch pseudometrics.

Lemma 2 Let $X$ be a compact metric space and $\rho$ be a compatible metric on $X$. Then the Besicovitch pseudometric and the pseudometric $D_{B}^{\prime}$ on $X^{\infty}$ given for $\underline{x}=\left\{x_{i}\right\}_{i=0}^{\infty}$ and $\underline{z}=\left\{z_{i}\right\}_{i=0}^{\infty}$ by

$$
D_{B}^{\prime}(\underline{x}, \underline{z})=\inf \left\{\delta>0: \bar{d}\left(\left\{n \geq 0: \rho\left(x_{n}, z_{n}\right) \geq \delta\right\}\right)<\delta\right\}
$$

are uniformly equivalent on $X^{\infty}$.

Proof Given $\underline{x}, \underline{z} \in X^{\infty}$ and $\delta>0$ we define

$$
J_{\delta}(\underline{x}, \underline{z})=\left\{n \geq 0: \rho\left(x_{n}, z_{n}\right) \geq \delta\right\} .
$$

Let $\chi_{\delta}$ denote the characteristic function of $J_{\delta}(\underline{x}, \underline{z})$. Recall that diam $X=1$, hence we have

$$
\delta \cdot \chi_{\delta}(n) \leq \rho\left(x_{n}, z_{n}\right) \leq \chi_{\delta}(n)+\delta .
$$

Summing the above from $n=0$ to $N-1$, dividing by $N$ and passing with $N$ to infinity we get

$$
\delta \cdot \bar{d}\left(J_{\delta}(\underline{x}, \underline{z})\right) \leq D_{B}(\underline{x}, \underline{z}) \leq \bar{d}\left(J_{\delta}(\underline{x}, \underline{z})\right)+\delta .
$$


Note also that

$$
D_{B}^{\prime}(\underline{x}, \underline{z}) \leq \varepsilon \text { if and only if } \bar{d}\left(J_{\varepsilon}(\underline{x}, \underline{z})\right)<\varepsilon .
$$

Fix $\varepsilon>0$. We claim that if $\delta<\varepsilon / 2$, then $D_{B}^{\prime}(\underline{x}, \underline{z})<\delta$ implies $D_{B}(\underline{x}, \underline{z})<\varepsilon$. To prove the claim assume that $D_{B}^{\prime}(\underline{x}, \underline{z})<\varepsilon / 2$ and use (2) to see that the right hand side of (1) is strictly smaller than $\varepsilon$. This proves our claim and yields uniform continuity of id: $\left(X^{\infty}, D_{B}^{\prime}\right) \rightarrow\left(X^{\infty}, D_{B}\right)$.

We now show that id: $\left(X^{\infty}, D_{B}\right) \rightarrow\left(X^{\infty}, D_{B}^{\prime}\right)$ is uniformly continuous. Fix $\varepsilon>0$ and $\delta<\varepsilon^{2}$. Take any pair $\underline{x}, \underline{z} \in X^{\infty}$ such that $D_{B}(\underline{x}, \underline{z})<\delta$. Then by the first inequality in (1) and the definition of $\delta$ we see that

$$
\varepsilon \cdot \bar{d}\left(J_{\varepsilon}(\underline{x}, \underline{z})\right) \leq D_{B}(\underline{x}, \underline{z})<\varepsilon^{2} .
$$

Therefore $\bar{d}\left(J_{\varepsilon}(\underline{x}, \underline{z})\right)<\varepsilon$, which implies that $D_{B}^{\prime}(\underline{x}, \underline{z}) \leq \varepsilon$ by (2).

Lemma 2 yields immediately the following corollary (for a proof it is enough to note that if $\rho$ and $\tilde{\rho}$ are uniformly equivalent, then so are $D_{B}^{\prime}$ and $\tilde{D}_{B}^{\prime}$ ).

Corollary 3 Let $\tilde{\rho}$ be another compatible metric on $X$ and $\tilde{D}_{B}$ and $\tilde{D}_{B}^{\prime}$ be defined as above with $\tilde{\rho}$ in place of $\rho$. Then $\tilde{D}_{B}$ and $D_{B}$ are uniformly equivalent on $X^{\infty}$.

We will now prove that if $(X, \sigma)$ is a shift space (symbolic dynamical system), then the Besicovitch pseudometric is uniformly equivalent with the $\bar{d}$-pseudometric measuring the upper asymptotic density of the set of coordinates at which two symbolic sequences differ. This is probably a folklore, but for completeness we present a proof inspired by [13].

Let $(X, T)$ be a dynamical systems and $x \in X$. We define $\underline{x}_{T}$ to be the sequence $x, T(x), T^{2}(x), \ldots \in X^{\infty}$. Given a continuous function $f: X \rightarrow \mathbb{R}$ we define

$$
f\left(\underline{x}_{T}\right)=\left(f(x), f(T(x)), \ldots, f\left(T^{n}(x)\right), \ldots\right) \in \mathbb{R}^{\infty}
$$

and for any family $\mathcal{F}$ containing continuous functions from $X$ to $\mathbb{R}$ we set

$$
D_{B}^{\mathcal{F}}\left(x, x^{\prime}\right)=\sup _{f \in \mathcal{F}} D_{B}\left(f\left(\underline{x}_{T}\right), f\left(\underline{x}_{T}^{\prime}\right)\right) .
$$

Theorem 4 Let $(X, T)$ be a dynamical system and let $\mathcal{F}$ be a uniformy equicontinuous and uniformly bounded family of real-valued functions on $X$ such that $\mathcal{F}_{T}=\left\{f \circ T^{j}\right.$ : $f \in \mathcal{F}, j \geq 0\}$ separates the points of $X$. Then the pseudometrics $D_{B}$ and $D_{B}^{\mathcal{F}}$ are uniformly equivalent on $X$.

Proof Using compactness it is easy to see that if the family $\mathcal{F}_{T}$ separates the points of $X$, then there exists a sequence $\left\{f_{n}\right\}_{n=1}^{\infty} \subset \mathcal{F}_{T}$ separating the points of $X$, where $f_{n}=g_{n} \circ T^{k(n)}$ for some $k(n) \geq 0$ and $g_{n} \in \mathcal{F}$. Let $x, x^{\prime} \in X$ and $n \geq 1$. Define $z=T^{k(n)}(x)$ and $z^{\prime}=T^{k(n)}\left(x^{\prime}\right)$. Note that

$$
D_{B}\left(f_{n}\left(\underline{x}_{T}\right), f_{n}\left(\underline{x}_{T}^{\prime}\right)\right)=D_{B}\left(g_{n}\left(\underline{z}_{T}\right), g_{n}\left(\underline{z}_{T}^{\prime}\right)\right)=D_{B}\left(g_{n}\left(\underline{x}_{T}\right), g_{n}\left(\underline{x}_{T}^{\prime}\right)\right) .
$$


Therefore

$$
\begin{aligned}
\sup _{n \geq 1} D_{B}\left(f_{n}\left(\underline{x}_{T}\right), f_{n}\left(\underline{x}_{T}^{\prime}\right)\right) & =\sup _{n \geq 1} D_{B}\left(g_{n}\left(\underline{x}_{T}\right), g_{n}\left(\underline{x}_{T}^{\prime}\right)\right) \\
& \leq D_{B}^{\mathcal{F}}\left(x, x^{\prime}\right)=\sup _{f \in \mathcal{F}} D_{B}\left(f\left(\underline{x}_{T}\right), f\left(\underline{x}_{T}^{\prime}\right)\right) .
\end{aligned}
$$

Take any sequence of weights $\left\{a_{n}\right\}_{n=1}^{\infty} \subset$ such that

$$
0<a_{n}<1 \text { and } \sum_{n=1}^{\infty} a_{n}\left\|f_{n}\right\|_{\infty}<\infty,
$$

where $\|\cdot\|_{\infty}$ denotes the sup norm. For $y, z \in X$ define

$$
\rho^{\prime}(y, z)=\sum_{n=1}^{\infty} a_{n}\left|f_{n}(y)-f_{n}(z)\right| .
$$

Note that $\rho^{\prime}$ is a metric on $X$ equivalent to $\rho$ because $f_{n}$ 's are equicontinuous and separate the points of $X$.

Fix $\varepsilon>0$. Find $m \in \mathbb{N}$ such that

$$
\sum_{n=m+1}^{\infty} a_{n}\left\|f_{n}\right\|_{\infty}<\varepsilon / 2 .
$$

Let $x, x^{\prime} \in X$ be such that $D_{B}^{\mathcal{F}}\left(x, x^{\prime}\right)<\varepsilon /(2 m)$. By Corollary 3 the Besicovitch pseudometric $D_{B}^{\prime}$ associated with $\rho^{\prime}$ is uniformly equivalent to $D_{B}$. Therefore it is enough to check that $D_{B}^{\prime}\left(x, x^{\prime}\right)<\varepsilon$. We have

$$
\begin{aligned}
D_{B}^{\prime}\left(x, x^{\prime}\right) & =\limsup _{N \rightarrow \infty} \frac{1}{N} \sum_{i=0}^{N-1} \rho^{\prime}\left(T^{i}(x), T^{i}\left(x^{\prime}\right)\right) \\
& =\limsup _{N \rightarrow \infty} \frac{1}{N} \sum_{i=0}^{N-1} \sum_{n=1}^{\infty} a_{n}\left|f_{n}\left(T^{i}(x)\right)-f_{n}\left(T^{i}\left(x^{\prime}\right)\right)\right| \\
& \leq \limsup _{N \rightarrow \infty} \frac{1}{N} \sum_{i=0}^{N-1} \sum_{n=1}^{m}\left(a_{n}\left|f_{n}\left(T^{i}(x)\right)-f_{n}\left(T^{i}\left(x^{\prime}\right)\right)\right|+\varepsilon / 2\right) \\
& =\varepsilon / 2+\limsup _{N \rightarrow \infty} \frac{1}{N} \sum_{i=0}^{N-1} \sum_{n=1}^{m} a_{n}\left|f_{n}\left(T^{i}(x)\right)-f_{n}\left(T^{i}\left(x^{\prime}\right)\right)\right| \\
& \leq \varepsilon / 2+\sum_{n=1}^{m} a_{n}\left(\limsup _{N \rightarrow \infty} \frac{1}{N} \sum_{i=0}^{N-1}\left|f_{n}\left(T^{i}(x)\right)-f_{n}\left(T^{i}\left(x^{\prime}\right)\right)\right|\right)<\varepsilon .
\end{aligned}
$$

The last inequality holds because we took $x, x^{\prime} \in X$ with $D_{B}^{\mathcal{F}}\left(x, x^{\prime}\right)<\varepsilon /(2 m)$. This proves that 


$$
\text { id: }\left(X, D_{B}^{\mathcal{F}}\right) \rightarrow\left(X, D_{B}\right)
$$

is uniformly continuous.

To prove that

$$
\text { id: }\left(X, D_{B}\right) \rightarrow\left(X, D_{B}^{\mathcal{F}}\right)
$$

is uniformly continuous one can follow the reasoning as in the proof of Lemma 2 to conclude that thanks to the uniform boundedness of $\mathcal{F}$ it is enough to show that for every $\varepsilon>0$ there exists $\delta>0$ such that if $x, x^{\prime} \in X$ satisfy $D_{B}\left(x, x^{\prime}\right)<\delta$ then for every $f \in \mathcal{F}$ we have

$$
\bar{d}\left(\left\{n: \rho\left(f\left(T^{n}(x)\right), f\left(T^{n}\left(x^{\prime}\right)\right)\right) \geq \varepsilon\right\}\right)<\varepsilon .
$$

The latter easily follows from uniform equicontinuity of $\mathcal{F}$ and Lemma 2.

Observe that any finite family $\mathcal{F}$ is uniformly equicontinuous and uniformly bounded. In particular, if $X$ is the space of sequences over a finite alphabet and $T$ is the shift map, then taking $\mathcal{F}=\left\{\iota_{0}\right\}$, where $\iota_{0}\left(x_{0} x_{1} x_{2} \ldots\right)=x_{0}$ yields the following corollary.

Corollary 5 If $\mathcal{A}$ is a finite set with discrete topology, then the Besicovitch pseudometric associated with any admissible metric on the product space $\mathcal{A}^{\infty}$ and the $\bar{d}$ -pseudometric on $\mathcal{A}^{\infty}$ given by

$$
\bar{d}\left(x, x^{\prime}\right)=\bar{d}\left(\left\{n \geq 0: x_{n} \neq x_{n}^{\prime}\right\}\right)=\limsup _{n \rightarrow \infty} \frac{\#\left\{0 \leq j<n: x_{j} \neq x_{j}^{\prime}\right\}}{n}
$$

are uniformly equivalent.

\section{Besicovitch pseudometric and invariant measures}

We are going to show that the map $\underline{x} \mapsto \hat{\omega}(\underline{x})$ is a uniformly continuous map from $X^{\infty}$ equipped with $D_{B}$ to the space of nonempty compact subsets of $\mathcal{M}(X)$ equipped with the Hausdorff metric.

Lemma 6 For every $\varepsilon>0$ there is $\delta>0$ such that if $\underline{x}, \underline{x}^{\prime} \in X^{\infty}$ and $D_{B}\left(\underline{x}, \underline{x}^{\prime}\right)<\delta$, then $\vec{d}\left(\mathfrak{m}(\underline{x}, n), \mathfrak{m}\left(\underline{x}^{\prime}, n\right)\right) \leq \varepsilon$ for all sufficiently large $n$.

Proof Fix $\varepsilon>0$. By Lemma 2 there exist $\delta>0$ such that if $\underline{x}, \underline{x}^{\prime} \in X^{\infty}$ and $D_{B}\left(\underline{x}, \underline{x}^{\prime}\right)<\delta$, then one can find $N \in \mathbb{N}$ such that for every $n \geq N$ one has

$$
\#\left\{0 \leq j<n: \rho\left(x_{j}, x_{j}^{\prime}\right) \geq \varepsilon\right\}<n \cdot \varepsilon .
$$

Take $n \geq N$ and a Borel set $B \subset X$. We have

$$
\mathfrak{m}(\underline{x}, n)(B)=\frac{1}{n} \#\left\{0 \leq j<n: x_{j} \in B\right\} \leq \mathfrak{m}\left(\underline{x}^{\prime}, n\right)\left(B^{\varepsilon}\right)+\varepsilon .
$$

Hence $\vec{d}\left(\mathfrak{m}(\underline{x}, n), \mathfrak{m}\left(\underline{x}^{\prime}, n\right)\right) \leq \varepsilon$ for all $n \geq N$. 
Theorem 7 For every $\varepsilon>0$ there is $\delta>0$ such that for all $\underline{x}, \underline{x}^{\prime} \in X^{\infty}$ with $D_{B}\left(\underline{x}, \underline{x}^{\prime}\right)<\delta$ one has $H_{\mathcal{M}(X)}\left(\hat{\omega}(\underline{x}), \hat{\omega}\left(\underline{x}^{\prime}\right)\right)<\varepsilon$.

Proof Fix $\varepsilon>0$. Use Lemma 6 to find $\delta$ for $\varepsilon / 3$. Take $\underline{x}, \underline{x}^{\prime} \in X^{\infty}$ such that $D_{B}\left(\underline{x}, \underline{x}^{\prime}\right)<\delta$. Let $\mu \in \hat{\omega}(\underline{x})$. Then $\mu=\lim _{k \rightarrow \infty}\left\{\mathfrak{m}\left(\underline{x}, n_{k}\right)\right\}_{k=0}^{\infty}$ for some strictly increasing sequence $\left\{n_{k}\right\}_{k=0}^{\infty} \in \mathbb{N}^{\infty}$. Let $v$ be an accumulation point of the sequence $\left\{\mathfrak{m}\left(\underline{x}^{\prime}, n_{k}\right)\right\}_{k=0}^{\infty}$. Taking a subsequence we can assume that

$$
\lim _{k \rightarrow \infty}\left\{\mathfrak{m}\left(\underline{x}^{\prime}, n_{k}\right)\right\}_{k=0}^{\infty}=v .
$$

By Lemma 6 we have $\vec{d}\left(\mathfrak{m}\left(\underline{x}, n_{k}\right), \mathfrak{m}\left(\underline{x}^{\prime}, n_{k}\right)\right) \leq \varepsilon / 3$ provided that $k$ is large enough. Let $k$ be sufficiently large to guarantee also that $\vec{d}\left(\mu, \mathfrak{m}\left(\underline{x}, n_{k}\right)\right)<\varepsilon / 3$ and $\vec{d}\left(v, \mathfrak{m}\left(\underline{x}^{\prime}, n_{k}\right)\right)<\varepsilon / 3$. Consequently,

$$
\vec{d}(\mu, v) \leq \vec{d}\left(\mu, \mathfrak{m}\left(\underline{x}, n_{k}\right)\right)+\vec{d}\left(\mathfrak{m}\left(\underline{x}, n_{k}\right), \mathfrak{m}\left(\underline{x^{\prime}}, n_{k}\right)\right)+\vec{d}\left(\mathfrak{m}\left(\underline{x}^{\prime}, n_{k}\right), v\right)<\varepsilon
$$

Hence we have

$$
\operatorname{dist}\left(\mu, \hat{\omega}\left(\underline{x}^{\prime}\right)\right)=\min \left\{\vec{d}(\mu, v): v \in \hat{\omega}\left(\underline{x}^{\prime}\right)\right\}<\varepsilon .
$$

Therefore $\max \left\{\operatorname{dist}\left(\mu, \hat{\omega}\left(\underline{x}^{\prime}\right)\right): \mu \in \hat{\omega}(\underline{x})\right\}<\varepsilon$. By the same argument we also have $\max \left\{\operatorname{dist}(v, \hat{\omega}(\underline{x})): v \in \hat{\omega}\left(\underline{x}^{\prime}\right)\right\}<\varepsilon$ and hence $H_{\mathcal{M}(X)}\left(\hat{\omega}(\underline{x}), \hat{\omega}\left(\underline{x}^{\prime}\right)\right)<\varepsilon$.

Corollary 8 Let $\underline{x}, \underline{x}^{\prime} \in X^{\infty}$. If $D_{B}\left(\underline{x}, \underline{x}^{\prime}\right)=0$, then $\hat{\omega}(\underline{x})=\hat{\omega}\left(\underline{x}^{\prime}\right)$.

Remark 9 It is easy to see that if $\underline{x}=\left\{x_{n}\right\}_{n=0}^{\infty} \in X^{\infty}$ is a $\delta$-average-pseudo-orbit for $T$, then $D_{B}(T(\underline{x}), \sigma(\underline{x}))<\delta$.

For any continuous map $T: X \rightarrow X$ let $\hat{T}: \mathcal{M}(X) \rightarrow \mathcal{M}(X)$ be given by the formula $\hat{T}(\mu)=\mu \circ T^{-1}$. The proof of the following fact can be found in [9, Proposition 3.2] (see also [35, Theorem 6.7]).

Theorem 10 The map $\hat{T}: \mathcal{M}(X) \rightarrow \mathcal{M}(X)$ is continuous. Additionally, $\hat{T}$ is surjective if and only if $T$ is surjective.

Corollary 11 For every $\varepsilon>0$ there is $\delta>0$ such that if $\underline{x}=\left\{x_{n}\right\}_{n=0}^{\infty} \in X^{\infty}$ fulfills $D_{B}(T(\underline{x}), \sigma(\underline{x}))<\delta$, then for every $\mu \in \hat{\omega}(\underline{x})$ one has $\vec{d}(\mu, \hat{T}(\mu))<\varepsilon$.

Proof Let $\mu \in \hat{\omega}(\underline{x})$ and $\mathfrak{m}\left(\underline{x}, k_{n}\right) \rightarrow \mu$ as $n \rightarrow \infty$, where $\left\{k_{n}\right\}_{n=0}^{\infty}$ is a strictly increasing sequence of nonnegative integers. For every Borel set $B$ and every $x \in X$ one has $\hat{\delta}(x)\left(T^{-1}(B)\right)=\hat{\delta}(T(x))(B)$. Consequently,

$$
\begin{aligned}
\hat{T}(\mathfrak{m}(\underline{x}, n))(B)=\mathfrak{m}(\underline{x}, n)\left(T^{-1}(B)\right) & =\frac{1}{n} \sum_{j=0}^{n-1} \hat{\delta}\left(x_{j}\right)\left(T^{-1}(B)\right) \\
& =\frac{1}{n} \sum_{j=0}^{n-1} \hat{\delta}\left(T\left(x_{j}\right)\right)(B)=\mathfrak{m}(T(\underline{x}), n)(B) .
\end{aligned}
$$


Because of the continuity of $\hat{T}$ one has

$$
\hat{T}(\mu)=\hat{T}\left(\lim _{n \rightarrow \infty} \mathfrak{m}\left(\underline{x}, k_{n}\right)\right)=\lim _{n \rightarrow \infty} \hat{T}\left(\mathfrak{m}\left(\underline{x}, k_{n}\right)\right)=\lim _{n \rightarrow \infty} \mathfrak{m}\left(T(\underline{x}), k_{n}\right) .
$$

Note that $\vec{d}(\mathfrak{m}(\underline{x}, n), \mathfrak{m}(\sigma(\underline{x}), n)) \leq 2 / n$ holds for every $n \in \mathbb{N}$. Hence

$$
\mu=\lim _{n \rightarrow \infty} \mathfrak{m}\left(\sigma(\underline{x}), k_{n}\right) .
$$

To finish the proof fix $\varepsilon>0$ and use Lemma 6 to pick $\delta>0$ for $\varepsilon / 2$. Then $D_{B}(\sigma(\underline{x}), T(\underline{x}))<\delta$ implies $\vec{d}\left(\mathfrak{m}\left(\sigma(\underline{x}), k_{n}\right), \mathfrak{m}\left(T(\underline{x}), k_{n}\right)\right) \leq \varepsilon / 2$ for all sufficiently large $n$. Therefore $\vec{d}(\mu, \hat{T}(\mu))<\varepsilon$ by (3) and (4).

Remark 12 A sequence $\underline{x}=\left\{x_{n}\right\}_{n=0}^{\infty}$ is an asymptotic average pseudo orbit if and only if $D_{B}(T(\underline{x}), \sigma(\underline{x}))=0$.

Corollary 13 If $\underline{x}=\left\{x_{n}\right\}_{n=0}^{\infty} \in X^{\infty}$ is an asymptotic average pseudo orbit, then $\hat{\omega}(\underline{x}) \subset \mathcal{M}_{T}(X)$.

Proof Since $D_{B}(T(\underline{x}), \sigma(\underline{x}))=0$ it follows from Corollary 11 that for every $\varepsilon>0$ and every $\mu \in \hat{\omega}(\underline{x})$ one has $\vec{d}(\mu, \hat{T}(\mu)) \leq \varepsilon$. Hence $\mu=\hat{T}(\mu)$ and so $\mu \in \mathcal{M}_{T}(X)$.

We finish this section with a yet another application of the Besicovitch pseudometric. Although we will not use this result we add it here since we belive it is of independent interest. Note that Weiss [37] proves the analog of Theorem 15 for symbolic system and convergence with respect to the $\bar{d}$-pseudometric. By Corollary 5 Weiss' result follows from our theorem.

Theorem 14 ([29], Sect. 5) Let $x \in X$ be generic for some $\mu \in \mathcal{M}_{T}(X)$. Then the following conditions are equivalent:

(1) $\mu \in \mathcal{M}_{T}^{e}(X)$,

(2) For all $f \in \mathcal{C}(X)$ and $\alpha>0$

$$
\bar{d}\left(\left\{n:\left|\frac{1}{k} \sum_{j=0}^{k-1} f\left(T^{n+j}(x)\right)-\int_{X} f d \mu\right|>\alpha\right\}\right) \rightarrow 0 \text { as } k \rightarrow \infty
$$

Theorem 15 Let $\left\{\mu_{p}\right\}_{p=1}^{\infty} \subset \mathcal{M}_{T}^{e}(X)$ and $\left\{x_{p}\right\}_{p=1}^{\infty} \subset X^{\infty}$ be such that $x_{p}$ is a generic point for $\mu_{p}$ for every $p \in \mathbb{N}$. If $x \in X$ is such that $D_{B}\left(x_{p}, x\right) \rightarrow 0$ as $p \rightarrow \infty$, then $x$ is generic for some $\mu \in \mathcal{M}_{T}^{e}(X)$.

Proof By Theorem 7 the function $\left(X^{\infty}, D_{B}\right) \ni \underline{x} \rightarrow \hat{\omega}(\underline{x}) \in\left(\mathcal{M}(X), H_{\mathcal{M}(X)}\right)$ is continuous, and so there exists $\mu \in \mathcal{M}_{T}(X)$ such that $\mu_{p} \rightarrow \mu$ as $p \rightarrow \infty$ and $x \in \operatorname{Gen}(\mu)$. We will show that $\mu \in \mathcal{M}_{T}^{e}(X)$. To this end we are going to show that 
the condition from Theorem 14(2) is fulfilled. Fix $f \in \mathcal{C}(X)$. Notice that for every $k, n, p \in \mathbb{N}$ we have

$$
\begin{aligned}
\left|\frac{1}{k} \sum_{j=0}^{k-1} f\left(T^{n+j}(x)\right)-\int_{X} f \mathrm{~d} \mu\right| \leq & \frac{1}{k} \sum_{j=0}^{k-1} f\left(T^{n+j}(x)\right)-\frac{1}{k} \sum_{j=0}^{k-1} f\left(T^{n+j}\left(x_{p}\right)\right) \mid \\
& +\left|\frac{1}{k} \sum_{j=0}^{k-1} f\left(T^{n+j}\left(x_{p}\right)\right)-\int_{X} f \mathrm{~d} \mu_{p}\right| \\
& +\left|\int_{X} f \mathrm{~d} \mu_{p}-\int_{X} f \mathrm{~d} \mu\right| .
\end{aligned}
$$

Take any $\alpha>0$. Let $P^{\prime} \in \mathbb{N}$ be such that for every $p \geq P^{\prime}$ we have

$$
\left|\int_{X} f \mathrm{~d} \mu_{p}-\int_{X} f \mathrm{~d} \mu\right| \leq \alpha / 3
$$

Then for every $p \geq P^{\prime}$ and any $k$ we have

$$
\begin{aligned}
& \left\{n \in \mathbb{N}_{0}:\left|\frac{1}{k} \sum_{j=0}^{k-1} f\left(T^{n+j}(x)\right)-\int_{X} f \mathrm{~d} \mu\right|>\alpha\right\} \\
& \subset\left\{n \in \mathbb{N}_{0}: \frac{1}{k} \sum_{j=0}^{k-1}\left|f\left(T^{n+j}(x)\right)-f\left(T^{n+j}\left(x_{p}\right)\right)\right|>\alpha / 3\right\} \\
& \cup\left\{n \in \mathbb{N}_{0}:\left|\frac{1}{k} \sum_{j=0}^{k-1} f\left(T^{n+j}\left(x_{p}\right)\right)-\int_{X} f \mathrm{~d} \mu_{p}\right|>\alpha / 3\right\} .
\end{aligned}
$$

Therefore it is enough to prove that for any $\varepsilon>0$ we can find $p \geq P^{\prime}$ and $K \in \mathbb{N}$ such that for all $k \geq K$ the following inequalities hold

$$
\begin{aligned}
& \bar{d}\left(\left\{n \in \mathbb{N}_{0}: \frac{1}{k} \sum_{j=0}^{k-1}\left|f\left(T^{n+j}(x)\right)-f\left(T^{n+j}\left(x_{p}\right)\right)\right|>\alpha / 3\right\}\right)<\varepsilon / 2, \\
& \bar{d}\left(\left\{n \in \mathbb{N}_{0}:\left|\frac{1}{k} \sum_{j=0}^{k-1} f\left(T^{n+j}\left(x_{p}\right)\right)-\int_{X} f \mathrm{~d} \mu_{p}\right|>\alpha / 3\right\}\right)<\varepsilon / 2 .
\end{aligned}
$$

Note that the second inequality above holds for each $p$ and all sufficiently large $k$ by Theorem 14. So assume that the first inequality fails for some parameter $\varepsilon$, that is, there exists $\varepsilon_{0}>0$ such that for all $p \geq P^{\prime}$ and every $K \in \mathbb{N}$ there is $k_{0} \geq K$ with

$$
\bar{d}\left(\left\{n \in \mathbb{N}_{0}: \frac{1}{k_{0}} \sum_{j=0}^{k_{0}-1}\left|f\left(T^{n+j}(x)\right)-f\left(T^{n+j}\left(x_{p}\right)\right)\right|>\alpha / 3\right\}\right) \geq \varepsilon_{0} / 2 .
$$


Let $\delta>0$ be such that $x^{\prime}, x^{\prime \prime} \in X$ and $\rho\left(x^{\prime}, x^{\prime \prime}\right)<\delta$ imply $\left|f\left(x^{\prime}\right)-f\left(x^{\prime \prime}\right)\right|<\alpha / 6$. We are going to show that (5) implies that there is a constant $\gamma>0$ such that for all $p \geq P^{\prime}$ we have

$$
\bar{d}\left(\left\{n \in \mathbb{N}_{0}: \rho\left(T^{n}(x), T^{n}\left(x_{p}\right)\right) \geq \delta\right\}\right) \geq \gamma
$$

But the inequality (6) cannot hold for all $p$, because it contradicts $D_{B}\left(x_{p}, x\right) \rightarrow 0$ as $p \rightarrow \infty$. Hence it remains to prove that (5) implies (6). Let $k_{0}$ be one of those $k$ 's for which (5) holds and let

$$
B(n)=\left\{0 \leq m<n: \frac{1}{k_{0}} \sum_{j=0}^{k_{0}-1}\left|f\left(T^{m+j}(x)\right)-f\left(T^{m+j}\left(x_{p}\right)\right)\right|>\alpha / 3\right\} .
$$

It follows from (5) that for each $N_{0}$ there exists $n \geq N_{0}$ such that $\# B(n) \geq n \varepsilon_{0} / 4$. For each $m \in B(n)$ it is easy to see that

$$
\#\left\{0 \leq j<k_{0}:\left|f\left(T^{m+j}(x)\right)-f\left(T^{m+j}\left(x_{p}\right)\right)\right|>\alpha / 6\right\} \geq \frac{\alpha k_{0}}{12\|f\|_{\infty}} .
$$

Therefore for infinitely many $n$ we have

$$
\#\left\{0 \leq m<n+k_{0}: \rho\left(T^{m}(x), T^{m}\left(x_{p}\right)\right)>\delta\right\} \geq \frac{\# B(n)}{k_{0}} \cdot \frac{\alpha k_{0}}{12\|f\|_{\infty}} \geq \frac{n \varepsilon_{0} \alpha}{48\|f\|_{\infty}} .
$$

But the above inequality clearly yields (6).

\section{Weak specification implies the asymptotic average shadowing property}

In [19] it was proved that a surjective system with the almost specification property satisfies the asymptotic average shadowing property. On the other hand it follows from [23] that the notions of almost specification and weak specification are independent, that is there exist: a system with the weak specification property which does not have the almost specification property and a system satisfying the almost specification property without the weak specification property. In this section we will show that weak specification also implies the asymptotic average shadowing property.

Recall that $g: \mathbb{N} \times\left(0, \varepsilon_{0}\right) \rightarrow \mathbb{N}_{0}$, where $\varepsilon_{0}>0$, is a mistake function if for all $\varepsilon<\varepsilon_{0}$ and all $n \in \mathbb{N}$ we have $g(n, \varepsilon) \leq g(n+1, \varepsilon)$ and $g(n, \varepsilon) / n \rightarrow 0$ as $n \rightarrow \infty$. Given a mistake function $g$ we define a function $k_{g}:(0, \infty) \rightarrow \mathbb{N}$ by $k_{g}(\varepsilon)=\min \{n \in \mathbb{N}: g(m, \varepsilon)<m \varepsilon$ for all $m \geq n\}$. For $0<\varepsilon<\varepsilon_{0}$ and $n \geq k_{g}(\varepsilon)$ large enough for the inequality $g(n, \varepsilon)<n$ to hold we set

$$
I(g ; n, \varepsilon):=\{\Lambda \subset\{0,1, \ldots, n-1\}: \# \Lambda \geq n-g(n, \varepsilon)\} .
$$


Given $x \in X, \varepsilon>0, n \in \mathbb{N}$ and a mistake function $g$ we define a Bowen ball by

$$
B_{n}(x, \varepsilon)=\left\{y \in X: \rho\left(T^{j}(x), T^{j}(y)\right)<\varepsilon \text { for every } 0 \leq j<n\right\}
$$

and a Bowen ball with mistakes by

$$
B_{n}(g ; x, \varepsilon)=\left\{y \in X:\left\{0 \leq j<n: \rho\left(T^{j}(x), T^{j}(y)\right)<\varepsilon\right\} \in I(g, n, \varepsilon)\right\} .
$$

We also need the following:

Lemma 16 ([22], Theorem 17) Let $(X, T)$ be a dynamical system. If $T$ is surjective and has the weak specification property, then $T$ is mixing.

Lemma 17 Let $(X, T)$ be a surjective dynamical system with the weak specification property and $M_{\varepsilon}(n)$ is the constant associated to $\varepsilon>0$ and $n \in \mathbb{N}$ in the definition of the weak specification property. Assume we are given

(1) an increasing sequence of integers $\left\{\alpha_{j}\right\}_{j=1}^{\infty} \subset \mathbb{N}$ with $\alpha_{1}=1$,

(2) a sequence of positive real numbers $\left\{\varepsilon_{i}\right\}_{i=1}^{\infty}$ such that for every $i \in \mathbb{N}$ one has

$$
\varepsilon_{\alpha_{i}}=\varepsilon_{\alpha_{i}+1}=\cdots=\varepsilon_{\alpha_{i+1}-1}=2 \cdot \varepsilon_{\alpha_{i+1}},
$$

(3) a sequence of points $\left\{x_{i}\right\}_{i=1}^{\infty} \subset X$,

(4) a sequence of integers $\left\{n_{i}\right\}_{i=0}^{\infty}$ satisfying $n_{0}=0, M_{\varepsilon_{i} / 2}\left(n_{i}\right) \leq n_{i}$ and

$$
n_{\alpha_{i}}=n_{\alpha_{i}+1}=\cdots=n_{\alpha_{i+1}-1} \text { for any } i \in \mathbb{N} \text {. }
$$

Then, setting

$$
l_{j}=\sum_{s=0}^{j-1} n_{s} \text { for } j \in \mathbb{N}
$$

we can find $z \in X$ such that for every $j \in \mathbb{N}$ we have

$$
T^{l_{j}}(z) \in \overline{B_{n_{j}}\left(g ; x_{j}, \varepsilon_{j}\right)},
$$

where $g: \mathbb{N} \times(0,1) \rightarrow \mathbb{N}$ is given by $g(n, \varepsilon)=M_{\varepsilon / 2}(n)$.

Proof We will define inductively a sequence $\left\{z_{n}\right\}_{n=1}^{\infty} \subset X$ such that for every $i \in \mathbb{N}$, every $s=1,2, \ldots, i$ and every $j \in\left\{\alpha_{s}, \ldots, \alpha_{s+1}-1\right\}$ we have

$$
T^{l_{j}+M_{\varepsilon_{j} / 2}\left(n_{j}\right)}\left(z_{i}\right) \in B_{n_{j}-M_{\varepsilon_{j} / 2}\left(n_{j}\right)}\left(T^{M_{\varepsilon_{j} / 2}\left(n_{j}\right)}\left(x_{j}\right), \varepsilon_{j} \cdot\left(1-1 / 2^{i-s+1}\right)\right) .
$$

Observe that if we put $m=k+M_{\varepsilon}(k)$ for some $k \in \mathbb{N}$ and $\varepsilon>0$ then $M_{\varepsilon}(m) \geq M_{\varepsilon}(k)$. It follows that (7) implies that

$$
T^{l_{j}}\left(z_{i}\right) \in B_{n_{j}}\left(g ; x_{j}, \varepsilon_{j} \cdot\left(1-1 / 2^{i-s+1}\right)\right) \subset \overline{B_{n_{j}}\left(g ; x_{j}, \varepsilon_{j}\right)} .
$$


We will now define $z_{1}$. Take $j \in\left\{1,2, \ldots, \alpha_{2}-1\right\}$ and note that if we set $a_{j}=$ $l_{j-1}+M_{\varepsilon_{1} / 2}\left(n_{j}\right)$ and $b_{j}=l_{j-1}+n_{j}$, then

$$
\left\{T^{\left[a_{j}, b_{j}\right]}\left(x_{j}\right): j=1,2, \ldots, \alpha_{2}-1\right\}
$$

is an $M_{\varepsilon_{1} / 2}\left(n_{1}\right)$-spaced specification. By the weak specification property, there exists $z_{1}$ satisfying

$$
T^{l_{j}}\left(z_{1}\right) \in B_{n_{j}-M_{\varepsilon_{1} / 2}\left(n_{j}\right)}\left(T^{M_{\varepsilon_{1} / 2}\left(n_{j}\right)}\left(x_{j}\right), \varepsilon_{1} \cdot(1-1 / 2)\right)
$$

for each $j=1, \ldots, \alpha_{2}-1$. Indeed (7) holds for $i=1$.

Assume that we have constructed a point $z_{k}$ satisfying (7). Then the sequence

$$
\left\{T^{\left[0, \alpha_{k}\right]}\left(z_{k}\right)\right\} \cup\left\{T^{\left[a_{j}, b_{j}\right]}\left(x_{j}\right): j=\alpha_{k+1}, \ldots, \alpha_{k+2}-1\right\}
$$

where $a_{j}=l_{j-1}+M_{\varepsilon_{j} / 2}\left(n_{j}\right)$ and $b_{j}=l_{j-1}+n_{j}$ for $j=\alpha_{k+1}, \ldots, \alpha_{k+2}-1$ is an $M_{\varepsilon_{\alpha_{k+1}} / 2}\left(n_{\alpha_{k+1}}\right)$-spaced specification. Using the weak specification property we find a point $z_{k+1}$ which $\varepsilon_{\alpha_{k+1}} / 2$-traces the specification given by (9). Observe that for $s=1, \ldots, k$ and $j=\alpha_{s}, \ldots, \alpha_{s+1}-1$ we have

$$
\begin{aligned}
\varepsilon_{j} \cdot\left(1-1 / 2^{k-s+1}\right)+\varepsilon_{\alpha_{k+1}} / 2 & =\varepsilon_{j} \cdot\left(1-1 / 2^{k-s+2}\right)-\frac{\varepsilon_{\alpha_{s}}}{2^{k-s+2}}+\varepsilon_{\alpha_{k+1}} / 2 \\
& =\varepsilon_{j} \cdot\left(1-1 / 2^{(k+1)-s+1}\right)-\frac{2^{k+1-s} \varepsilon_{\alpha_{k+1}}}{2^{k-s+2}}+\varepsilon_{\alpha_{k+1}} / 2 \\
& =\varepsilon_{j} \cdot\left(1-1 / 2^{(k+1)-s+1}\right)
\end{aligned}
$$

hence (7) is satisfied also with $i=k+1$. Finally note that $d\left(z_{n}, z_{n+1}\right)<\varepsilon_{\alpha_{n+1}}$ for every $n$, hence $\left\{z_{n}\right\}_{n=1}^{\infty}$ is a Cauchy sequence. Putting $z=\lim _{n \rightarrow \infty} z_{n}$ ends the proof by (8).

The next theorem is an analog of [19, Theorem 3.5]. Although the weak specification property and the almost specification property are independent the proof follows the same lines.

Theorem 18 If a surjective dynamical system $(X, T)$ has the weak specification property, then it has the asymptotic average shadowing property.

Proof The proof [19, Theorem 3.5] relies on chain mixing of every surjective dynamical system with the almost specification property ([19, Lemma 3.2]) and [19, Lemma 3.4]. The latter result is analogous to Lemma 17 with the only difference, that the almost specification property is replaced by the weak specification property. Therefore the Theorem follows from the proof of [19, Theorem 3.5] with Lemma 16 and 17 used where [19, Lemma 3.2] and [19, Lemma 3.4] were applied in [19].

Remark 19 In fact, it was proved in [38, Corollary 6.9] that there is no need to assume that $T$ is surjective in [19, Theorem 3.5]. We do not know whether the surjectivity assumption can be relaxed in Theorem 18 . 


\section{Asymptotic average pseudo-orbits and periodic decompositions}

Following Banks [3] we say that a collection $\mathcal{D}=\left\{D_{0}, \ldots, D_{n-1}\right\}$ of subsets of $X$ is a regular periodic decomposition if the following conditions are satisfied:

(1) $T\left(D_{i}\right) \subset D_{(i+1) \bmod n}$ for $0 \leq i<n$,

(2) $D_{i}=\overline{\operatorname{int}\left(D_{i}\right)}$ for each $0 \leq i<n$,

(3) the intersection $D_{i} \cap D_{j}$ is nowhere dense whenever $i \neq j$,

(4) $\bigcup_{i=0}^{n-1} D_{i}=X$.

Before we state the main result of this section we make the following observation. We leave the proof to the reader.

Remark 20 Fix $r>0$. Let $\left\{n_{k}\right\}_{k=0}^{\infty} \subset \mathbb{N}$ be such that $0=n_{0}<n_{1}<n_{2}<\ldots$ and $n_{k}=0(\bmod r)$. Then $\bar{d}\left(\left\{s_{N}: N \in \mathbb{N}\right\}\right)=0$, where $s_{N}=\sum_{j=0}^{N} n_{j}$. If $\underline{z}=\left\{z_{j}\right\}_{j=0}^{\infty}$ is given by $z_{k}=T^{k-s_{N}}\left(y_{N}\right)$ for $s_{N} \leq k<s_{N+1}$, where $\left\{y_{N}\right\}_{N=0}^{\infty} \subset X$, then:

(1) The sequence $\underline{z}=\left\{z_{n}\right\}_{n=0}^{\infty}$ is an average asymptotic pseudo orbit for $T$ and $\underline{z}^{\prime}=\left\{z_{n r}\right\}_{n=0}^{\infty}$ is an average asymptotic pseudo orbit for $T^{r}$.

(2) If $x \in X$ is such that $D_{B}\left(\underline{z}^{\prime}, \underline{x}_{T^{r}}\right)=0$, then $D_{B}\left(\underline{z}, \underline{x}_{T}\right)=0$.

Now we are ready to prove the following.

Theorem 21 Assume that $(X, T)$ is a dynamical system with a regular periodic decomposition $\left\{D_{0}, \ldots, D_{r-1}\right\}$ and that $\left(D_{0}, T^{r}\right)$ has the asymptotic average shadowing property. If $V \subset \mathcal{M}_{\mathrm{T}}(X)$ is nonempty, closed and connected, then there is a point $x \in X$ such that $\hat{\omega}(x)=V$. In particular, every $T$-invariant measure has a generic point.

Proof Let $V$ be a nonempty, closed and connected subset of $\mathcal{M}_{\mathrm{T}}(X)$. Sigmund (see [34, Remark 1]) proved that there is an asymptotic average pseudoorbit $\underline{z}=\left\{z_{n}\right\}_{n=0}^{\infty}$ for $T$ such that $\hat{\omega}(z)=V$ (note that Sigmund used different terminology). It is easy to see that actually [34, Remark 1] allows us to assume that $z_{k}=T^{k-s_{N}}\left(y_{N}\right)$ for $s_{N} \leq k<s_{N+1}$ for some sequences $\left\{n_{k}\right\}_{k=0}^{\infty} \subset \mathbb{N}$ and $\left\{y_{N}\right\}_{N=0}^{\infty} \subset D_{0}$ such that $0=n_{0}<n_{1}<n_{2}<\cdots$ and $n_{k}=0(\bmod r)$. By Remark 20 the sequence $z^{\prime}=$ $\left\{z_{n r}\right\}_{n=0}^{\infty}$ is an average asymptotic pseudo-orbit for $T^{r}$ and furthermore, by definition we obtain that also $\underline{z}^{\prime} \subset D_{0}$. Let $x \in D_{0}$ asymptotically trace on average $z^{\prime}$ for $T^{r}$. Then $D_{B}\left(\underline{z}^{\prime}, \underline{x}_{T^{r}}\right)=0$ and using Remark 20 one more time we get $D_{B}\left(\underline{z}, \underline{x}_{T}\right)=0$. By Corollary 8 we have $\hat{\omega}(x)=\hat{\omega}(\underline{z})=V$ which ends the proof.

Since it is proved in [19] that the almost specification property implies the average shadowing property and in Sect. 5 we have shown that the same follows from the weak specification property, Theorem 21 immediately implies the following corollary, which can be also found in [10] (with a different proof).

Corollary 22 If $(X, T)$ is surjective and has the relative weak specification property (or relative almost specification property), then for every asymptotic average pseudo orbit $\underline{x}=\left\{x_{i}\right\}_{i=0}^{\infty} \in X^{\infty}$ there exists a point $z$ such that $\hat{\omega}(\underline{x})=\hat{\omega}(z)$. In particular, if $V \subset \mathcal{M}_{\mathrm{T}}(X)$ is nonempty, closed and connected, then there is a point $z \in X$ such that $\hat{\omega}(z)=V$ and every $T$-invariant measure has a generic point. 
Corollary 22 extends results from [7,8,31-34].

Example 23 The following example shows that there are maps which do not have the asymptotic average shadowing property but have a regular periodic decomposition $\left\{D_{0}, \ldots, D_{r-1}\right\}$ such that $\left.T^{r}\right|_{D_{0}}$ satisfies the asymptotic average shadowing property.

By $\mathbb{T}^{k}=\mathbb{R}^{k} / \mathbb{Z}^{k}$ we denote the $k$-dimensional torus. Let $S: \mathbb{T}^{k} \rightarrow \mathbb{T}^{k}$ be a toral automorphism whose associated linear map is nonhyperbolic but has no roots of unity as eigenvalues. It was proved in $[24,26]$ that such an automorphism satisfies the weak specification property. Let $n \geq 2$ and $T_{n}: \mathbb{T}^{1} \rightarrow \mathbb{T}^{1}$ (see Fig. 1) be given by

$$
T_{n}(x)= \begin{cases}3 x+1 / n, & \text { if } 0 \leq x<1 /(3 n) \\ -3 x+3 / n, & \text { if } 1 /(3 n) \leq x<2 /(3 n), \\ 3 x-1 / n, & \text { if } 2 /(3 n) \leq x<1 / n \\ x+1 / n \quad \bmod 1, & \text { if } 1 / n \leq x \leq 1\end{cases}
$$

Define $F_{n}=S \times T_{n}: \mathbb{T}^{k+1} \rightarrow \mathbb{T}^{k+1}$. Notice that

$$
\left\{\mathbb{T}^{k} \times[0,1 / n], \mathbb{T}^{k} \times[1 / n, 2 / n], \ldots, \mathbb{T}^{k} \times[(n-1) / n, 1]\right\}
$$

is a regular periodic decomposition for $F_{n}$. Moreover, $\left.F_{n}^{n}\right|_{\mathbb{T}^{k} \times[0,1 / n]}$ is a product of two maps which have the weak specification property and hence satisfies it as well. To see that $F_{n}$ does not have the asymptotic average shadowing property notice that the Lebesgue measure is a fully supported invariant measure for $F_{n}$. By [19, Theorems 3.7 $\& 4.3]$ any map with the asymptotic average shadowing and fully supported invariant

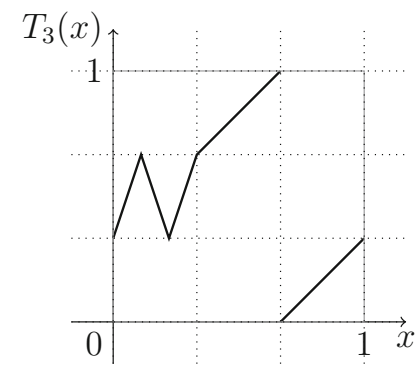

Fig. 1 Graph of the map $T_{3}$

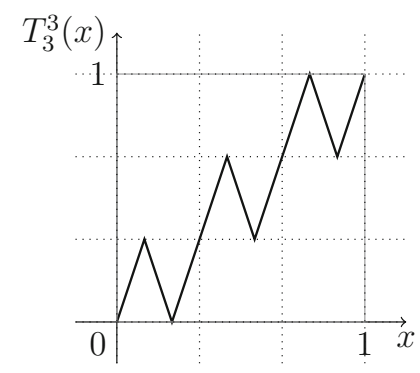

Fig. 2 Graph of the map $T_{3}^{3}$ 


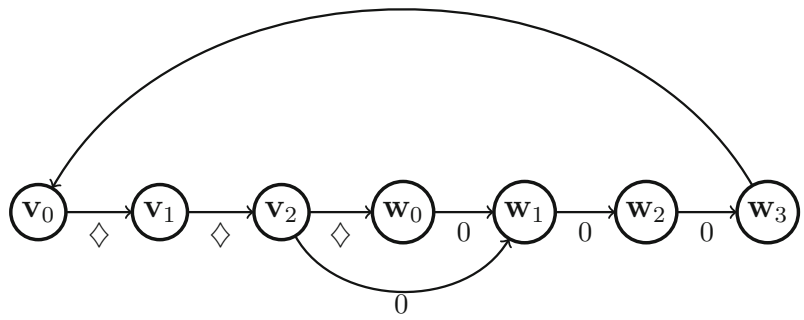

Fig. 3 The graph $\mathcal{G}^{(4,6)}$

measure must be weakly mixing, but $F_{n}^{n}$ is not even transitive as $F_{n}^{n}\left(\mathbb{T}^{k} \times[0,1 / n]\right) \subset$ $\mathbb{T}^{k} \times[0,1 / n]$ (see Fig. 2).

\section{Shift space with the asymptotic shadowing property but without almost specification}

In this section we show that the proximal dynamical system $\mathbb{Y}$ with positive entropy introduced in [27] has the average shadowing property and fully supported invariant measure. By [23, Theorem 5.3], a nontrivial proximal system like $\mathbb{Y}$ never has the almost specification property. Recall that a pair $(x, y) \in X \times X$ is proximal, if

$$
\liminf _{n \rightarrow \infty} \rho\left(T^{n}(x), T^{n}(y)\right)=0 .
$$

A dynamical system is proximal if every pair $(x, y) \in X \times X$ is proximal. It remains open whether $\mathbb{Y}$ has the asymptotic average shadowing property.

We recall the construction from [27]. Let $\mathcal{A}=\{0, \diamond\}$ and let $1<s<t$ be positive integers. We define a labelled graph $\mathcal{G}^{(s, t)}=(V, E, i, t, \mathcal{L})$ over $\mathcal{A}$ in the following way (for the definition of labelled graph and sofic shift see [25]):

$$
\begin{aligned}
& V=\left\{v_{0}, \ldots, v_{t-s}, w_{0}, \ldots, w_{s-1}\right\}, \quad E=\left\{e_{1}, \ldots, e_{t-s}, e_{1}^{\prime}, \ldots, e_{s-1}^{\prime}, \hat{e}_{1}, \hat{e}_{2}, \hat{e}_{3}\right\}, \\
& i(e)=\left\{\begin{array}{ll}
v_{i-1}, & e=e_{i} \\
w_{i-1}, & e=e_{i}^{\prime} \\
w_{s-1}, & e=\hat{e}_{3} \\
v_{t-s}, & e \in\left\{\hat{e}_{1}, \hat{e}_{2}\right\},
\end{array} \quad t(e)= \begin{cases}v_{i}, & e=e_{i} \\
w_{i}, & e=e_{i}^{\prime} \\
v_{0}, & e=\hat{e}_{3} \\
w_{0}, & e=\hat{e}_{1} \\
w_{1}, & e=\hat{e}_{2},\end{cases} \right. \\
& \mathcal{L}(e)= \begin{cases}\diamond, & e=e_{i} \text { or } e=\hat{e}_{1} \\
0, & e=e_{i}^{\prime} \text { or } e \in\left\{\hat{e}_{2}, \hat{e}_{3}\right\} .\end{cases}
\end{aligned}
$$

Denote by $X_{(s, t)}$ the shift space consisting of sequences over $\mathcal{A}$ which are labels of infinite paths on $\mathcal{G}^{(s, t)}$. In other words, $x \in X_{(s, t)}$ is a concatenation of blocks of $s$ zeros separated by blocks of $t-s$ or $t-s+1$ symbols $\diamond$ (Fig. 3). Denote by $p^{(s, t)}$ a periodic sequence of the form $\left(\diamond^{t-s} 0^{s}\right)^{\infty} \in \mathcal{A}^{\infty}$. Now, define the following subsets of $\{0,1\}^{\infty}$ : 


$$
\begin{aligned}
& F^{(s, t)}=\left\{x \in\{0,1\}^{\infty}: \text { there exists } y \in X_{(s, t)} \text { such that if } y_{i}=0, \text { then } x_{i}=0\right\}, \\
& P^{(s, t)}=\left\{x \in\{0,1\}^{\infty}: \text { if } p_{i}^{(s, t)}=0, \text { then } x_{i}=0\right\} .
\end{aligned}
$$

It is easy to verify that each $F^{(s, t)}$ is a shift in $\{0,1\}^{\infty}$.

Let $s_{i}=2^{i}$ and $t_{i}=10^{i}$. Define

$$
\mathbb{Y}=\bigcap_{i=1}^{\infty} F^{\left(s_{i}, t_{i}\right)}
$$

Note that

$$
t_{i}>3 s_{i}+2 i>5 i \quad \text { and } \quad 0<\sum_{i=1}^{\infty} \frac{s_{i}}{t_{i}}=\frac{1}{4}<1 .
$$

It was proved in [27] that (10) implies that $\mathbb{Y}$ is a topologically mixing and proximal system with positive topological entropy. Let

$$
Y_{n}=X^{\left(s_{1}, t_{1}\right)} \cap \ldots \cap X^{\left(s_{n}, t_{n}\right)} .
$$

Lemma 24 For every $\varepsilon>0$ there is $n>0$ such that if $x \in Y_{n}$, then there is $z \in \mathbb{Y}$ with $\bar{d}(x, z)<\varepsilon$.

Proof Fix $\varepsilon>0$. Use (10) to find $n>0$ such that

$$
\sum_{i=n+1}^{\infty} \frac{s_{i}}{t_{i}}<\varepsilon
$$

Take $x \in Y_{n}$. Define $z$ by $z_{j}=0$ if there is $i>n$ such that $p_{j}^{\left(s_{i}, t_{i}\right)}=0$, and $z_{j}=x_{j}$ otherwise. Then $z \in \mathbb{Y}$ and $\bar{d}(x, z)<\varepsilon$.

Lemma 25 Let $X$ be a shift space. Iffor every $\varepsilon>0$ there is a shift space $X_{\varepsilon}$ with the average shadowing property such that $X \subset X_{\varepsilon}$ and for every $x \in X_{\varepsilon}$ there is $z \in X$ with $\bar{d}(x, z)<\varepsilon$, then $X$ has the average shadowing property.

Proof Fix $\varepsilon>0$. Let $\beta>0$ be such that $\bar{d}\left(x, x^{\prime}\right)<\beta$ implies $D_{B}\left(x, x^{\prime}\right)<\varepsilon / 2$ (the existence of such a $\beta$ is guaranteed by Corollary 5). Consider shift space $X_{\beta}$. Use the average shadowing property for $X_{\beta}$ to find $\delta>0$ such that every $\delta$-average pseudoorbit for $\sigma$ contained in $X_{\beta}$ is $\varepsilon / 2$ traced on average. Let $\underline{x}$ be a $\delta$-average pseudoorbit for $\sigma$ contained in $X$. Since $X \subset X_{\beta}$ there is a point $y \in X_{\beta}$ which $\varepsilon / 2$ traces $\underline{x}$ in average. Use the assumption to find a point $z \in X$ such that $\bar{d}(y, z)<\beta$. Hence $D_{B}\left(\underline{z}_{T}, \underline{x}\right) \leq D_{B}(z, y)+D_{B}\left(\underline{y}_{T}, \underline{x}\right)<\varepsilon$.

Lemma 26 For every nonempty open set $U \subset \mathbb{Y}$ there is $y \in \mathbb{Y}$ such that $N(y, U)$ has positive upper density.

Proof Let $z \in U$ and $k$ be such that the cylinder set defined by $z_{[0, k)}$ is contained in $U$. Take any $n>k$ such that $t_{n}-s_{n}>k$ and 


$$
1-k \sum_{r=n+1}^{\infty} \frac{s_{r}}{t_{r}}:=\gamma>0
$$

Then the periodic point $x=\left(z_{[0, k)} 0^{t_{n}-k}\right)^{\infty}$ belongs to $Z=\bigcap_{j=1}^{n} F^{\left(s_{i}, t_{i}\right)}$ and $t_{n} \mathbb{N} \subset$ $\left\{i: x_{[i, i+k)}=z_{[0, k)}\right\}$. Define $p \in\{0, \diamond\}^{\infty}$ by $p_{j}=0$ if there is $i>n$ such that $p_{j}^{\left(s_{i}, t_{i}\right)}=0$, and $p_{j}=\diamond$ otherwise. Then $p \in \bigcap_{i=n+1}^{\infty} P^{\left(s_{i}, t_{i}\right)}$ and we claim that the set $A=\left\{i: p_{[i, i+k)}=\diamond^{k}\right\}$ has positive upper density. To see this, note that

$$
d\left(\left\{i: p_{i}=0\right\}\right) \leq \sum_{r=n+1}^{\infty} \frac{s_{r}}{t_{r}},
$$

and since $p_{i}=0$ and $i \geq k$ implies $p_{[i-l, i-l+k)} \neq \diamond^{k}$ for $l=-k+1,-k+2, \ldots, 0$ we have

$$
\bar{d}(A) \geq 1-k d\left(\left\{i: p_{i}=0\right\}\right) \geq \gamma .
$$

We have $\bigcup_{j=0}^{t_{n}-1} j+t_{n} \mathbb{N}=\mathbb{N}$, therefore for some $j \in\left\{0,1, \ldots, t_{n}-1\right\}$ the set $A_{j}=(A+j) \cap t_{n} \mathbb{N}$ has upper density at least $\gamma / t_{n}$. For that $j$ we construct a point $y=y_{0} y_{1} y_{2} \ldots$ by

$$
y_{i}= \begin{cases}x_{i}, & i \in A_{j}+[0, k), \\ 0, & \text { otherwise }\end{cases}
$$

then $y \in \mathbb{Y}$ and obviously by our construction we have that $A_{j} \subset N(y, U)$, hence $N(y, U)$ has positive upper density.

Theorem 27 The shift space $\mathbb{Y}$ is mixing, has the average shadowing property, positive topological entropy and fully supported invariant measure.

Proof Proximality, positive topological entropy and topological mixing follows from [27]. Let $\mathcal{U}=\left\{U_{n}: n \in \mathbb{N}\right\}$ be the base for the topology on $\mathbb{Y}$. Lemma 26 implies that for any open set $U_{n} \in \mathcal{U}$ we can find an invariant measure $\mu_{n}$ such that $\mu(U)>0$ (e.g. see [16]). Then $\mu=\sum_{n=1}^{\infty} 2^{-n} \mu_{n}$ is a fully supported measure.

Note that $Z_{N}=\bigcap_{i=1}^{N} F^{\left(s_{i}, t_{i}\right)}$ is the intersection of mixing sofic shifts for every $N \in \mathbb{N}$, therefore $Z_{N}$ is a mixing sofic shift. Since every mixing sofic shift has the specification property, it follows from [20] that $Z_{N}$ has the asymptotic average shadowing property and so it also has the average shadowing property by [19]. Now $\mathbb{Y}$ has the average shadowing property by Lemmas 24 and 25.

Corollary 28 The subshift $\mathbb{Y}$ has the average shadowing property but does not have the almost specification property.

Proof By [23] minimal points are dense in the measure center of any dynamical system with the almost specification property. On the other hand, it follows from Theorem 27 
that $\mathbb{Y}$ is a nontrivial (hence uncountable) proximal system whose measure center equals the whole space. Proximality implies that $\mathbb{Y}$ has a fixed point which is the unique minimal point in the system (e.g. see [1]). Therefore minimal points of $\mathbb{Y}$ can not be dense in the measure center and $\mathbb{Y}$ can not have the almost specification property.

Our last example shows that the assumptions of Lemma 25 can not be weakened.

Given a shift space $X$ over an alphabet $\mathcal{A}$ we define the finite type approximation of order $m \in \mathbb{N}$ of $X$ by

$$
X_{m}=\left\{x \in \mathcal{A}^{\infty}: \forall k \in \mathbb{N}_{0} \exists y \in X x_{[k, k+m]}=y_{[0, m]}\right\}
$$

In other words $X_{m}$ is a shift of finite type determined by finite blocks of length $m+1$ occurring in $X$ (see [9, p.111]). The following lemma is probably well-known (cf. Proposition 3.62 in [21]).

Lemma 29 Let $X$ be a mixing shift space. The following conditions are equivalent:

(1) $X$ is chain mixing,

(2) $X_{m}$ is mixing for every $m \in \mathbb{N}$.

Sketch of the proof Note that for every $m$ the points in $X_{m}$ are in the one to one correspondence with $\delta_{m}$-chains in $X$, where $\delta_{m}$ goes to 0 as $m \rightarrow \infty$. To see this take a point $x \in X_{m}$ and for each $j \in \mathbb{N}_{0}$ pick $x^{(j)}$ in the cylinder in $X$ determined by $x_{[j, j+m]}$. Furthermore, chain mixing is equivalent to mixing for systems with the shadowing property and all shifts of finite type have the shadowing property by [36].

Corollary 30 Let $X$ be a mixing shift space. Then there exists a decreasing sequence $X_{0} \supset X_{1} \supset \cdots \supset X$ of mixing shifts of finite type such that $X=\bigcap_{n=1}^{\infty} X_{n}$.

Note that if $X=\bigcap_{n=1}^{\infty} X_{n}$ and $X_{1} \supset X_{2} \supset \cdots \supset X$ then for every $\varepsilon>0$ there is $n$ such that $\operatorname{dist}(x, X)<\varepsilon$ for each $x \in X_{n}$. Next Theorem shows that this is not enough to imply the average shadowing property on the intersection and the condition $\inf \left\{\bar{d}(x, y): x \in X, y \in X_{n}\right\}<\varepsilon$ for all $n$ large enough in Lemma 25 is essential.

Theorem 31 There exists a decreasing sequence $X_{1} \supset X_{2} \supset \cdots \supset X$ of mixing sofic shifts such that the shift space $X=\bigcap_{n=1}^{\infty} X_{n}$ is mixing but does not have the average shadowing property.

Proof Consider the shift space $X$ constructed in Sect. 7 of [14]. By [14, Lemmas 6-8] $X$ has the following properties:

(1) periodic points are dense,

(2) $X$ is mixing, nontrivial and $0^{\infty} \in X$,

(3) if $x \in X$ is not periodic then $d\left(\left\{i: x_{i}=1\right\}\right)=0$.

Since periodic points are dense and $X$ is nontrivial, there exists a periodic point $p \in X$ such that $d\left(\left\{i: p_{i}=1\right\}\right)=\alpha>0$. Using $p$ and $0^{\infty}$ interchangeably it is not hard to construct an asymptotic average pseudo-orbit $\left\{y^{(i)}\right\}_{i=0}^{\infty}$ such that 
(i) $\bar{d}\left(\left\{i: y_{0}^{(i)}=1\right\}\right)=\alpha>0$ and

(ii) $\bar{d}\left(\left\{i: y_{0}^{(i)}=0\right\}\right)=1$.

If $X$ has the average shadowing property, then by [38, Thm. 5.5] there exists $z \in X$ such that

$$
\underline{d}\left(\left\{i: z_{i}=y_{0}^{(i)}\right\}\right)>1-\alpha / 3
$$

This implies by (i) that $\bar{d}\left(\left\{i: z_{i}=1\right\}\right) \geq \alpha / 2$ and so $z$ must be a periodic point with $d\left(\left\{i: z_{i}=1\right\}\right)=\beta \geq \alpha / 2$. On the other hand by (ii) we see that $\bar{d}\left(\left\{i: z_{i}=0\right\}\right) \geq$ $1-\alpha / 3>1-\beta$ which is a contradiction. This shows that $X$ cannot have the average shadowing property. The proof is finished by Corollary 30 .

Acknowledgements We would like to thank both the reviewers for their contribution and comments. Dominik Kwietniak was supported by the National Science Centre (NCN) under Grant 2013/08/A/ST1/ 00275 and partially supported by CAPES/Brazil Grant No. 88881.064927/2014-01. Martha Łącka was supported by Grant No. PSP: K/DSC/002690. Research of Piotr Oprocha was partly supported by the project "LQ1602 IT4Innovations excellence in science".

Open Access This article is distributed under the terms of the Creative Commons Attribution 4.0 International License (http://creativecommons.org/licenses/by/4.0/), which permits unrestricted use, distribution, and reproduction in any medium, provided you give appropriate credit to the original author(s) and the source, provide a link to the Creative Commons license, and indicate if changes were made.

\section{References}

1. Akin, E., Kolyada, S.: Li-Yorke sensitivity. Nonlinearity 16(4), 1421-1433 (2003)

2. Auslander, J.: Mean- $L$-stable systems. Ill. J. Math. 3, 566-579 (1959)

3. Banks, J.: Regular periodic decompositions for topologically transitive maps. Ergod. Theory Dyn. Syst. 17(3), 505-529 (1997)

4. Barreira, L., Schmeling, J.: Sets of "non-typical" points have full topological entropy and full Hausdorff dimension. Isr. J. Math. 116, 29-70 (2000)

5. Blanchard, F., Formenti, E., Kurka, P.: Cellular automata in the Cantor, Besicovitch, and Weyl topological spaces. Complex Syst. 11(2), 107-123 (1997)

6. Bowen, R.: Equilibrium states and the ergodic theory of Anosov diffeomorphisms. Lecture Notes in Mathematics, vol. 470. Springer, Berlin (2008)

7. Dateyama, M.: Invariant measures for homeomorphisms with weak specification. Tokyo J. Math. 4(2), 389-397 (1981)

8. Dateyama, M.: Invariant measures for homeomorphisms with almost weak specification. In: Probability Theory and Mathematical Statistics (Tbilisi, 1982), volume 1021 of Lecture Notes in Mathematics, pp. 93-96. Springer, Berlin (1983)

9. Denker, M., Grillenberger, C., Sigmund, K.: Ergodic theory on compact spaces. Lecture Notes in Mathematics, vol. 527. Springer, Berlin (1976)

10. Dong, Y., Tian, X., Yuan, X.: Ergodic properties of systems with asymptotic average shadowing property. J. Math. Anal. Appl. 432(1), 53-73 (2015)

11. Downarowicz, T.: Positive topological entropy implies chaos DC2. Proc. Am. Math. Soc. 142(1), 137-149 (2014)

12. Downarowicz, T., Glasner, E.: Isomorphic extensions and applications. Topol Methods Nonlinear Anal 48(1), 321-338 (2016)

13. Downarowicz, T., Iwanik, A.: Quasi-uniform convergence in compact dynamical systems. Stud. Math. 89(1), 11-25 (1988)

14. Falniowski, F., Kulczycki, M., Kwietniak, D., Li, J.: Two results on entropy, chaos and independence in symbolic dynamics. Discr. Contin. Dyn. Syst. Ser. B 20(10), 3487-3505 (2015) 
15. Fuhrmann, G., Gröger, M., Jäger, T.: Amorphic complexity. Nonlinearity 29(2), 528 (2016)

16. Furstenberg, H.: Recurrence in Ergodic Theory and Combinatorial Number Theory. Princeton University Press, Princeton, N.J. (1981)

17. Gu, R.: The asymptotic average shadowing property and transitivity. Nonlinear Anal. 67(6), 1680-1689 (2007)

18. Jacobs, K., Keane, M.: 0 - 1-Sequences of Toeplitz type. Z. Wahrscheinlichkeitstheorie und Verw. Gebiete 13, 123-131 (1969)

19. Kulczycki, M., Kwietniak, D., Oprocha, P.: On almost specification and average shadowing properties. Fund. Math. 224(3), 241-278 (2014)

20. Kulczycki, M., Oprocha, P.: Exploring the asymptotic average shadowing property. J. Differ. Equ. Appl. 16(10), 1131-1140 (2010)

21. Kurka, P.: Topological and Symbolic Dynamics. Cours Spécialisés [Specialized Courses], vol. 11. Société Mathématique de France, Paris (2003)

22. Kwietniak, D., Łącka, M., Oprocha, P.: A panorama of specification-like properties and their consequences. In: Dynamics and Numbers, Contemporary Mathematics, vol. 669, pp. 155-186, AMS (2016)

23. Kwietniak, D., Oprocha, P., Rams, M.: On entropy of dynamical systems with almost specification. Isr. J. Math. 213(1), 475-503 (2016)

24. Lind, D.A.: Dynamical properties of quasihyperbolic toral automorphisms. Ergod. Theory Dyn. Syst. 2(1), 49-68 (1982)

25. Lind, D., Marcus, B.: An Introduction to Symbolic Dynamics and Coding. Cambridge University Press, Cambridge (1995)

26. Marcus, B.: A note on periodic points for ergodic toral automorphisms. Monatsh. Math. 89(2), 121-129 (1980)

27. Oprocha, P.: Families, filters and chaos. Bull. Lond. Math. Soc. 42(4), 713-725 (2010)

28. Ornstein, D., Weiss, B.: Mean distality and tightness. In: Dynamical systems and related problems of geometry. Collected papers dedicated to the memory of Academician Andrei Andreevich Bolibrukh. Transl. from the Russian, pp. 295-302. Moscow: Maik Nauka/Interperiodika (2004)

29. Oxtoby, J.C.: Ergodic sets. Bull. Am. Math. Soc. 58, 116-136 (1952)

30. Shields, P.C.: The ergodic theory of discrete sample paths. Graduate Studies in Mathematics, vol. 13. American Mathematical Society, Providence, RI (1996)

31. Sigmund, K.: Generic properties of invariant measures for Axiom A diffeomorphisms. Invent. Math. 11, 99-109 (1970)

32. Sigmund, K.: On dynamical systems with the specification property. Trans. Am. Math. Soc. 190, 285-299 (1974)

33. Sigmund, K.: On the distribution of periodic points for $\beta$-shifts. Monatsh. Math. 82(3), 247-252 (1976)

34. Sigmund, K.: On minimal centers of attraction and generic points. J. Reine Angew. Math. 295, 72-79 (1977)

35. Walters, P.: An Introduction to Ergodic Theory. Graduate Texts in Mathematics, vol. 79. Springer, New York (1982)

36. Walters, P.: On the pseudo-orbit tracing property and its relationship to stability. In: The structure of attractors in dynamical systems (Proc. Conf., North Dakota State Univ., Fargo, N.D., 1977), volume 668 of Lecture Notes in Math., pp. 231-244. Springer, Berlin (1978)

37. Weiss, B.: Single orbit dynamics. In: CBMS Regional Conference Series in Mathematics, vol. 95. American Mathematical Society, Providence, RI (2000)

38. Wu, X., Oprocha, P., Chen, G.: On various definitions of shadowing with average error in tracing. Nonlinearity 39, 1942-1972 (2016) 